\title{
A relação entre características dos conselhos de administração e o risco de mercado de estatais listadas
}

\section{The relation between board attributes and risk on listed state-owned enterprises}

\section{Carolina Coletta}

Escola Superior de Agricultura "Luiz de Queiroz",

Universidade de São Paulo

email:ccoletta@usp.br

\section{Roberto Arruda de Souza Lima}

Escola Superior de Agricultura "Luiz de Queiroz", Universidade de São Paulo email: raslima@usp.br

\section{RESUMO}

O objetivo deste estudo é investigar a relação entre características dos conselhos de estatais brasileiras listadas e seu risco de mercado, mensurado pela volatilidade dos preços das ações. Os conselhos de administração são responsáveis por supervisionar o gerenciamento dos riscos e, no caso de estatais, acrescenta-se a necessidade de criar controles para prevenir a corrupção. Considerando que o conselho deve deliberar sobre decisões estratégicas que, inerentemente, apresentam riscos, sua atuação e características pode impactar a estabilidade da empresa. A partir de uma população de 31 estatais listadas, foram selecionadas aquelas com maior liquidez em bolsa, no período de 2015 a 2017. O tamanho do conselho apresenta relação positiva com o risco, enquanto a proporção de mulheres e a proporção de membros independentes apresentaram relações negativas com o risco. No entanto, observou-se que as relações foram não significativas, sugerindo possíveis interferências e falhas de governança nessas empresas.

Palavras-Chave: governança corporativa; conselho de administração; risco; empresas estatais.

\section{ABSTRACT}

Board of directors are considered the main mechanism of corporate governance. Such boards must overlook risk management and create controls to prevent corruption in state-owned enterprises. Considering that board of directors deliberate about strategic decisions that inherently present risks, its performance and attributes can impact the firm's stability. The main purpose of this study is to investigate the relation between listed state-owned enterprises board attributes and its risk, measured by stock volatility. The firms with higher market liquidity were considered from 2015 to 2017. Board size presents a positive relation with risk, while the presence of women and independent directors present a negative relation with risk. However, these relations are not statistically significant, which points out to possible interference and flaws in the corporate governance of these firms.

Key-words: corporate governance; board of directors; risk; state-owned enterprises. 


\section{INTRODUÇÃO}

A partir do desenvolvimento das organizações, notadamente, pela separação entre a propriedade e o controle das empresas (BERLE; MEANS, 1932), surge a necessidade de monitorar a gestão e minimizar custos decorrentes dos conflitos de interesses, que se estabelecem na relação de agência entre gestores e acionistas (JENSEN; MECKLIN, 1976). Neste contexto, os conselhos de administração emergem como o órgão máximo de controle das decisões gerenciais e do monitoramento dos executivos (FAMA; JENSEN, 1983).

Especialmente nas empresas de controle estatal, além da função de monitoramento e do processo de decisão, ressalta-se que os conselhos devem criar controles internos para prevenir a possibilidade de corrupção (OECD, 2015). As estatais apresentam grande relevância para o cenário brasileiro, uma vez que compõem parte considerável do Ibovespa, além de atuarem em setores estratégicos e de infraestrutura, com impacto direto sobre a sociedade (SILVEIRA, 2010). No entanto, embora tenha grande relevância econômica, a literatura sobre governança corporativa em estatais, no contexto de países emergentes, ainda é limitada, segundo Thompson, Alleyne e Charles-Soverall (2019).

Destaca-se, ainda, que os conselhos são responsáveis por assegurar que os riscos das empresas sejam identificados, supervisionando a gestão dos mesmos (IBGC, 2015b, OECD, 2015). A Organização para a Cooperação e Desenvolvimento Econômico (OECD, 2015) ressalta o papel de supervisão do gerenciamento dos riscos das empresas de modo que o conselho deve determinar os tipos e níveis de risco que a empresa poderá arcar para alcançar seus objetivos.

Sendo o principal mecanismo interno de governança corporativa, o conselho de administração proporciona melhores tomadas de decisão, com foco na maximização de valor (SILVEIRA, 2010). No entanto, uma vez que o conselho é o órgão máximo de controle das decisões (FAMA; JENSEN, 1983), cabe aos conselheiros deliberar sobre decisões estratégicas do negócio, o que naturalmente possui riscos envolvidos. Ademais, as decisões tomadas pelo conselho podem impactar na estabilidade da empresa. Nesse sentido, observa-se que a qualidade das contribuições e atu- ação dos conselheiros depende de como o conselho é formado (MATHEW; IBRAHIM; ARCHBOLD, 2016).

Em relação à composição do conselho, podem-se destacar aspectos como o número de membros, quantidade de conselheiros independentes e a expressividade da diversidade, especialmente de gênero, encontrada nesses órgãos de controle. Tais aspectos são alvo de recomendações específicas nos principais códigos de boas práticas de governança corporativa (IBGC, 2015b; OECD, 2015).

Em relação ao tamanho do conselho, existe o argumento de que conselhos maiores podem apresentar problemas de coordenação (JENSEN, 1993) e, por outro lado, o argumento de que conselhos maiores diminuem a tomada de decisões extremistas (CHENG, 2008). A questão da independência dos conselhos é de grande relevância, especialmente no contexto das empresas estatais. Há grande propensão de indicações políticas para o conselho das estatais, comprometendo o monitoramento dos executivos e a tomada de decisões de forma mais adequada (MUSACCHIO; LAZZARINI; AGUILERA, 2015). Já a discussão da diversidade de gênero nos conselhos vem ganhando maior destaque, ressaltando-se a criação de legislação específica sobre o tema em países europeus, o que lança luz à tal problemática no contexto dos conselhos (ADAMS; FERREIRA, 2009). No entanto, não se verifica um consenso na literatura sobre o impacto da maior proporção de mulheres nos conselhos (SILA, GONZALEZ E HAGENDORFF, 2016).

Diante do exposto, emerge o seguinte problema de pesquisa: características dos conselhos de administração - como tamanho e proporção de membros independentes e de mulheres - apresenta relação com o risco apresentado pelas sociedades de economia mista? Dessa forma, o presente estudo tem como objetivo investigar a relação entre características dos conselhos de administração de estatais brasileiras listadas e seu risco de mercado

\section{FUNDAMENTAÇÃO TEÓRICA}

A governança corporativa nas empresas de controle estatal pode ser utilizada como forma de minimizar a interferência política, impedindo que tais 
empresas sejam meios de ganhos políticos, em detrimento do retorno do capital investido, especialmente nas sociedades de economia mista (MUSACCHIO; LAZZARINI; AGUILERA, 2015). Em tais empresas, há o desafio de conciliar diferentes objetivos organizacionais, relativos às atividades de interesse público, concomitantemente ao objetivo de maximização de capital dos acionistas privados (MIRANDA; AMARAL, 2011).

Um dos principais mecanismos internos de governança corporativa é o conselho de administração. Considerado o órgão máximo do sistema de controle de decisões nas organizações, exerce o papel de monitoramento dos gestores (FAMA; JENSEN, 1983) a fim de evitar conflitos de agência no seu relacionamento com os acionistas (JENSEN; MECKLING, 1976). Considerando-se as empresas de controle estatal, além do processo de decisão e monitoramento, compete aos conselhos a criação e manutenção de controles internos, ética e medidas de compliance para prevenir a corrupção em tais organizações (OECD, 2015). De acordo com Thompson, Alleyne e Charles-Soverall (2019) ainda são poucos os estudos que exploram a governança corporativa em empresas estatais, especialmente em países emergentes. No contexto brasileiro, Coletta e Lima (2020) investigaram a relação entre a estrutura do conselho de administração de estatais brasileiras listadas entre 2002 e 2017. Os autores verificaram que uma melhor estrutura do conselho de administração está associada ao maior valor da empresa e melhor desempenho financeiro, uma vez que conflitos de agência são minimizados com o melhor funcionamento do conselho.

Ademais, compete ao conselho de administração estabelecer a política de gerenciamento de riscos da empresa, além de orientar a diretoria no processo de gerenciamento de tais riscos, incluindo os riscos de corrupção e fraudes (IBGC, 2015a). Os conselheiros devem deliberar sobre decisões estratégicas do negócio, o que naturalmente possui riscos envolvidos, de modo que tais decisões podem impactar na estabilidade da empresa. Dessa forma, caso o conselho não desempenhe suas funções adequadamente, a empresa poderá apresentar riscos de mercado, associados à alta volatilidade das ações, aumentando as chances de insolvência da empresa (MATHEW; IBRAHIM; ARCHBOLD, 2016).
Nesse sentido, Mathew, Ibrahim e Archbold (2018) apontam para a relação negativa entre a estrutura dos conselhos e medidas de risco - risco total, risco idiossincrático e volatilidade do retorno dos ativos - para uma amostra de 268 empresas britânicas entre 2005 e 2010. Por outro lado, Paiva, Oliveira e Peixoto (2015) encontraram relação positiva entre um índice relativo às boas práticas dos conselhos de administração e a variável de volatilidade das ações, para uma amostra de empresas listadas na B3, entre 2012 e 2013.

Os riscos, de modo geral, representam a incerteza relacionada aos retornos esperados ao se realizar um investimento. A volatilidade de um fluxo de retornos de determinado ativo é considerada, junto com a maturidade desse ativo, como um fator que determina seu grau de risco (ASSAF NETO, 2010). Por sua vez, o risco de mercado, segundo Matias (2007), considera os riscos de perdas financeiras devido às variações nas cotações de ativos negociados no mercado financeiro.

Diante dessa discussão, Mathew, Ibrahim e Archbold (2016) defendem que a qualidade das contribuições e atuação dos conselheiros depende de como o conselho é formado. Um importante determinante do desempenho das atividades do conselho encontra-se no número de membros que o compõem (YERMACK, 1996). O Programa Destaque em Governança de Estatais, da B3 (2017) exige entre sete a onze membros no conselho. No entanto, Jensen (1993) argumenta que conselhos com mais de sete ou oito membros são menos efetivos na avaliação e monitoramento do CEO, devido a problemas de coordenação. Por outro lado, Cheng (2008) afirma que tomadas de decisões extremistas diminuem com o aumento do tamanho do conselho. Tal autor verificou que empresas com conselhos maiores apresentam menor variabilidade no retorno mensal das ações, no retorno sobre o ativo (ROA) e $q$ de Tobin, além de apresentarem gastos menores com pesquisa e desenvolvimento, além de reestruturações menos frequentes.

No que tange à relação com risco, Mathew, Ibrahim e Archbold (2016) afirmam que a literatura apresenta evidências de uma relação inversa entre o número de membros do conselho e o risco apresentado pela empresa. Nesse sentido, Huang e Wang (2015) concluíram que, para uma amostra de 
empresas chinesas listadas - a qual apresenta proeminente propriedade estatal - conselhos menores estão associados a políticas empresariais mais arriscadas. Em tais empresas, o tamanho médio dos conselhos é de 9,4 membros. Em concordância com tal resultado, Mathew, Ibrahim e Archbold (2018) analisaram 268 empresas britânicas - com 8,95 membros, em média, no conselho - e concluíram que o menor risco está associado a conselhos maiores. De modo semelhante, Nakano e Nguyen (2012) concluíram, para uma amostra de empresas japonesas, que conselhos maiores estão associados com empresas com menor risco de falência. Tais autores ressaltam que a associação de conselhos maiores à menor propensão a tomar decisões de risco está relacionada com a dificuldade em atingir um consenso na decisão de grandes grupos. Diante do exposto, formula-se a seguinte hipótese:

\section{$H_{1}$ : O tamanho do conselho apresenta relação negativa} com o risco.

A independência do conselho também é assunto de grande importância na discussão da governança corporativa, especialmente em empresas estatais. Neste contexto, há uma facilidade de indicação de cargos, com base em critérios políticos. Dessa forma, membros indicados ao conselho, pelo governo, irão apresentar pouco incentivo para monitorar os executivos e tomar decisões de forma mais adequada (MUSACCHIO; LAZZARINI; AGUILERA, 2015).

Quando não existem vínculos que conectam membros do conselho a executivos ou acionistas, melhores decisões podem ser tomadas com vistas ao resultado de longo prazo (SILVEIRA, 2010), o que minimizaria tomadas de decisões prejudiciais à organização devido à pressão política presente, exemplificada em Shleifer e Vishny (1994). Entretanto, mesmo os conselheiros independentes podem esconder uma afiliação política indireta (MENOZZI; URTIAGA; VANNONI, 2012) e destruir valor para as empresas, uma vez que esse tipo de conselheiro seria pouco efetivo em sua atividade de monitoramento da gestão (SHI. XU; ZHANG, 2018).

Em relação a medidas de risco, Cheng (2008) não identificou relação significativa entre a porcentagem de conselheiros independentes e a variabilidade dos retornos mensais das ações. De modo semelhante, os resultados de Mathew, Ibrahim e Archbold (2016) apontam para uma relação negativa, porém não significativa, entre proporção de conselheiros não-executivos e risco total e volatilidade do retorno dos ativos. Os autores explicam a ausência de significância estatística pela possibilidade de membros independentes não terem informação suficiente para desafiar decisões tomadas pelos executivos no conselho. No entanto, os conselheiros independentes, de acordo com Cheng (2008, p. 162) contribuem para os conselhos de administração por meio de sua visão heterogênea, o que atua como forma de moderar as tomadas de decisão, e possibilita menor variabilidade de desempenho (CHENG, 2008, p. 162). Nesse sentido, formula-se a seguinte hipótese:

$H_{2}$ : A proporção de membros independentes no conselho é negativamente relacionada com o risco.

As melhores práticas de governança corporativa também reforçam a necessidade de promover a diversidade nos conselhos e diretoria. A diversidade de perfis que compõem o conselho deve ser estimulada, pois contribui com diferentes perspectivas e argumentos, que irão promover a tomada de decisão com maior qualidade (IBGC, 2015b). Nesse sentido, segundo Allini, Rossi e Hussainey (2016) a discussão da diversidade nos conselhos de administração considera os diferentes perfis dos membros - sendo que a diversidade de gênero é a mais discutida atualmente - e como tais diferenças afetam o processo de tomada de decisão.

Segundo Mathew, Ibrahim e Archbold (2016), a literatura apresenta, majoritariamente, evidências de que a diversidade de gênero promove melhor governança. Adams e Ferreira (2009) verificaram que a presença de mulheres é positivamente relacionada com a eficácia dos conselhos. Os autores explicam que as mulheres comparecem com maior frequência às reuniões, e que quanto maior a proporção de mulheres no conselho, também é maior o comparecimento dos homens, indicando monitoramento em tais condições.

Sila, Gonzalez e Hagendorff (2016) afirmam que houve uma pressão recente, sobre diversas firmas, para a promoção de maior diversidade de gênero 
em seus conselhos. Os autores ressaltam que não existe um entendimento consolidado a respeito do impacto - inclusive sobre a propensão ao risco - do maior número de mulheres nos conselhos. No entanto, estudos como os de Charness e Gneezy (2012) e Powell e Ansic (1997) apresentam evidências de que as mulheres têm menor propensão ao risco em decisões de investimento, em relação aos homens.

O estudo conduzido por Sila, Gonzalez e Hagendorff (2016), não apresenta evidências de que a presença de mulheres no conselho reduz o equity risk. Segundo os autores, um conselho com maior proporção de mulheres não pode ser considerado mais ou menos propenso a aceitar riscos, se comparado a um conselho com maior presença masculina. Dessa forma, a diversidade de gênero nos conselhos é promovida com vistas à equidade, e não por razões puramente econômicas, concluem os autores. No entanto, Bernile, Bhagwat e Yonker (2018) concluíram que maior diversidade no conselho está associada à menor volatilidade dos retornos das ações e melhor desempenho das empresas. Para tais autores, tal resultado ocorre porque a diversidade atua como um mecanismo moderador das decisões. Diante das evidências expostas na literatura - em relação à menor propensão das mulheres ao risco, bem como à diversidade de gênero promovendo melhor governança - considera-se a seguinte hipótese:

$H_{3}$ : A proporção de mulheres no conselho apresenta relação negativa com o risco.

\section{PROCEDIMENTOS METODOLÓGICOS}

A população do estudo envolve 31 estatais listadas na B3, no período de 2015 a 2017. Para compor a amostra, foram selecionadas as empresas cujas ações tiveram negociação em pelo menos $70 \%$ dos pregões, para cada ano considerado. Tal critério de liquidez é necessário para mensuração da variável de volatilidade do preço das ações. Os dados foram coletados na base de dados Economatica. Justifica-se o período pela possibilidade de captar mudanças endógenas na estrutura dos conselhos de administração das estatais, uma vez que, com a promulgação da Lei Federal 13.303 de 2016, as estatais seriam obrigadas a implementarem, até 2018, medidas específicas de governança corporativa (BRASIL, 2016). Desse modo, obteve-se uma amostra composta por 11 empresas para os anos de 2015 e 2016, e 12 empresas em 2017, totalizando 34 observações.

Para mensurar o risco de mercado, pela variável 'volatilidade', foram coletados os preços de fechamento diários das ações das empresas, para o período de 2015 a 2017, por meio da base de dados Economática. As séries dos preços de fechamento das ações que apresentaram missing values - devido à ausência de negociação durante cinco dias, ou menos, em qualquer intervalo - foram tratadas por meio da interpolação linear, realizada através do SPSS, em concordância com Araújo et al. (2004). Em relação aos dados das variáveis independentes, relativas às características dos conselhos de administração, foram coletados dados dos Formulários de Referência - especificamente, da seção "12. Assembleia e Administração" - enviados pelas empresas à Comissão de Valores Mobiliários (CVM).

Considerando que o objetivo do estudo é verificar se há relação entre características dos conselhos e o risco de mercado das empresas, faz-se necessário estabelecer as variáveis independentes, para mensurar tais características, e a variável dependente, para mensurar o risco. Determinam-se as seguintes variáveis independentes: tamanho do conselho (TAMC), proporção de mulheres no conselho (MUL) e proporção de conselheiros independentes (INDP). Ressalta-se que a variável relativa à independência dos membros considerou a declaração de independência apresentada nos Formulários de Referência enviados pelas empresas à CVM.

Como variável dependente, relativa ao risco de mercado, será considerada a volatilidade (VOL) do preço das ações das empresas da amostra. Adotam-se, também, as seguintes variáveis de controle: tamanho da empresa (TAMF), retorno sobre o ativo (ROA), grau de endividamento (DIV) e adesão aos Níveis Diferenciados de Governança Corporativa (NDGC) da B3. A definição das variáveis e os estudos precedentes que as utilizaram são apresentadas no Tabela 1 . As perspectivas teóricas, que fundamentam a inserção de tais variáveis no modelo, encontram-se na Tabela 2. 
Tabela 1 Definição das variáveis

\begin{tabular}{l|l|l|l}
\multicolumn{1}{c|}{ Variável } & Classificação & \multicolumn{1}{c|}{ Definição operacional } & \multicolumn{1}{c}{ Estudos Anteriores } \\
\hline Volatilidade (VOL) & Dependente & $\begin{array}{l}\text { Logaritmo natural da razão entre cotação de } \\
\text { fechamento do dia t e t-1 }\end{array}$ & $\begin{array}{l}\text { Paiva, Oliveira e Peixoto (2015), Lameira } \\
\text { (2012), Espejo e Clemente (2016), Bernile, } \\
\text { Bhagwat e Yonker (2018) }\end{array}$ \\
\hline $\begin{array}{l}\text { Tamanho do conselho } \\
\text { (TAMC) }\end{array}$ & Independente & $\begin{array}{l}\text { Logaritmo natural do número de membros } \\
\text { do conselho }\end{array}$ & $\begin{array}{l}\text { Cheng (2008), Nakano e Nguyen (2012), } \\
\text { Huang e Wang (2015), Mathew, Ibrahim e } \\
\text { Archbold (2016), Sila, Gonzalez e Hagendorff } \\
\text { (2016), Bernile, Bhagwat e Yonker (2018) }\end{array}$ \\
\hline $\begin{array}{l}\text { Diversidade de gênero } \\
\text { (MUL) }\end{array}$ & Independente & Porcentagem de mulheres no conselho & $\begin{array}{l}\text { Mathew, Ibrahim e Archbold (2016), Sila, } \\
\text { Gonzalez e Hagendorff (2016) }\end{array}$ \\
\hline $\begin{array}{l}\text { Independência dos mem- } \\
\text { bros (INDP) }\end{array}$ & Independente & Porcentagem de membros independentes. & $\begin{array}{l}\text { Cheng (2008), Mathew, Ibrahim e Archbold } \\
\text { (2016), Sila, Gonzalez e Hagendorff (2016), }\end{array}$ \\
\hline $\begin{array}{l}\text { Tamanho da empresa } \\
\text { (TAMF) }\end{array}$ & Controle & $\begin{array}{l}\text { Logaritmo natural do valor contábil do ativo } \\
\text { total }\end{array}$ & $\begin{array}{l}\text { Cheng (2008), Paiva, Oliveira e Peixoto } \\
\text { (2015), Bernile, Bhagwat e Yonker (2018) }\end{array}$ \\
\hline $\begin{array}{l}\text { Retorno sobre o ativo } \\
\text { (ROA) }\end{array}$ & Controle & $\begin{array}{l}\text { Razão entre lucro líquido e ativo total do } \\
\text { ano anterior }\end{array}$ & $\begin{array}{l}\text { Mathew, Ibrahim e Archbold (2016), Bernile, } \\
\text { Bhagwat e Yonker (2018) }\end{array}$ \\
\hline $\begin{array}{l}\text { Endividamento (DIV) } \\
\text { Adesão aos Níveis Dife- } \\
\text { renciados de Governança } \\
\text { Corporativa (NDGC) }\end{array}$ & Controle & $\begin{array}{l}\text { Controle } \\
\text { presa esteja listada nos NDGC; assume valor } \\
\text { zero, caso contrário. }\end{array}$ & $\begin{array}{l}\text { Silva, Nardi e Pimenta Junior (2012); Paiva, } \\
\text { Oliveira e Peixoto (2015) }\end{array}$ \\
\hline
\end{tabular}

Fonte: elaborado pelos autores

Tabela 2 Perspectiva teórica das variáveis

\begin{tabular}{|c|c|c|c|}
\hline Variável & Perspectiva teórica & Variável & Perspectiva teórica \\
\hline VOL & $\begin{array}{l}\text { "A volatilidade idiossincrática está associada ao risco } \\
\text { idiossincrático que é específico a um determinado } \\
\text { ativo" (COSTA; MAZZEU; COSTA JR, 2016, p. 228) }\end{array}$ & TAMF & $\begin{array}{l}\text { Empresas maiores são capazes de diversificar e realizar } \\
\text { maiores investimentos, de modo que mesmo um } \\
\text { investimento desfavorável pode não impactar a volati- } \\
\text { lidade das ações. Espera-se, portanto, que empresas } \\
\text { maiores apresentem menor risco (MATHEW, IBRAHIM } \\
\text { E ARCHBOLD, 2016, p. 245) }\end{array}$ \\
\hline TAMC & $\begin{array}{l}\text { Tomadas de decisões extremistas pelo conselho de } \\
\text { administração diminuem com o aumento do número } \\
\text { de membros (CHENG, 2008, p. 158) }\end{array}$ & $\mathrm{ROA}$ & $\begin{array}{l}\text { O desempenho prévio da empresa pode modificar o } \\
\text { comportamento atual em relação ao risco, em uma } \\
\text { possível tentativa de compensar o resultado anterior } \\
\text { (MATHEW, IBRAHIM E ARCHBOLD, 2016, p. 245) }\end{array}$ \\
\hline MUL & $\begin{array}{l}\text { Apesar de não haver consenso sobre o impacto da } \\
\text { presença de mulheres nos conselhos (SILA, GONZA- } \\
\text { LEZ E HAGENDORFF, 2016, p. 26), há evidências de } \\
\text { que as mulheres têm menor propensão ao risco em } \\
\text { decisões de investimento (CHARNESS; GNEEZY, 2012; } \\
\text { POWELL; ANSIC, 1997) }\end{array}$ & DIV & $\begin{array}{l}\text { A volatilidade dos retornos das ações tem uma forte } \\
\text { relação positiva com o grau de alavancagem (CHRIS- } \\
\text { TIE, 1982, p. 407) }\end{array}$ \\
\hline INDP & $\begin{array}{l}\text { Conselheiros independentes podem contribuir com } \\
\text { uma visão heterogênea dentro do conselho, mode- } \\
\text { rando as tomadas de decisão e possibilitando menor } \\
\text { variabilidade de desempenho (CHENG, 2008, p. 162) }\end{array}$ & NDGC & $\begin{array}{l}\text { Os NDGC objetivam estabelecer melhores práticas de } \\
\text { governança para reduzir a assimetria de informação e } \\
\text { oferecer maior segurança ao mercado (SILVA; NARDI; } \\
\text { PIMENTA JUNIOR, 2012, p. 223) }\end{array}$ \\
\hline
\end{tabular}

Fonte: elaborado pelos autores 
A partir das variáveis descritas na Tabela 1, temse o seguinte modelo econométrico:

$$
\begin{aligned}
\mathrm{VOL}_{\mathrm{i}}=\alpha & +\beta_{1} \mathrm{TAMC}_{\mathrm{i}}+\beta_{2} \mathrm{MUL}_{\mathrm{i}}+\beta_{3} \mathrm{INDP}_{\mathrm{i}}+ \\
& +\beta_{4} \mathrm{TAMF}_{\mathrm{i}}+\beta_{5} \mathrm{ROA}_{\mathrm{i}}+\beta_{6} \mathrm{DIV}_{\mathrm{i}}+\beta_{7} \mathrm{NDGC}_{\mathrm{i}}
\end{aligned}
$$

A partir de tal modelo, foi realizada uma regressão múltipla, a fim de estimar o efeito de atributos do conselho de administração no risco de mercado de estatais listadas. Tal método está em concordância com demais estudos que tratam a relação entre atributos do conselho e risco, tais como: Sila, Gonzalez e Hagendorff (2016), Mathew, Ibrahim e Archbold (2018), Bernile, Bhagwat e Yonker (2018). O método de estimação utilizado foi Mínimos Quadrados Ordinário (MQO).

\section{ANÁLISE DOS RESULTADOS}

Inicialmente, são apresentadas as estatísticas descritivas das variáveis na Tabela 3.

Para o período analisado, o tamanho médio do conselho das sociedades de economia mista da amostra foi de 8,64, número que se encontra dentro das recomendações do IBGC (2015b) de 5 a 11 membros, e da Lei 13.303 (BRASIL, 2016), que estabelece de 7 a 11 conselheiros. $\mathrm{O}$ tamanho mínimo de conselho, identificado na amostra, foi de 6 membros, enquanto o tamanho máximo foi 15 membros, este último divergindo das principais recomendações de boas práticas de governança corporativa.

Os resultados da Tabela 3 apontam a baixa diversidade de gênero encontrada nos conselhos de administração das sociedades de economia mista analisadas. Observa-se que a proporção média de mulheres nos conselhos das estatais analisadas foi de $9 \%$, no período. No âmbito da discussão da diversidade de gênero nos conselhos de estatais, $o$ IBGC (2020) apenas ressalta que a diversidade deve ser promovida, sem apresentar medidas mais concretas para as empresas promoverem a diversidade de gênero, não apenas no conselho, mas também em sua diretoria. Nesse sentido, não há uma recomendação de proporção desejável de mulheres nos conselhos.

No que tange à proporção de membros independentes, os conselhos das estatais analisadas apresentaram, em média, uma proporção de $30 \%$. Em número absolutos, a amostra apresentou uma média de 2,7 conselheiros independentes, sendo que uma das empresas não apresentou conselheiros declarados como independentes, e a maior proporção encontrada de foi de $77,8 \%$. A proporção média verificada em tais empresas está de acordo com a B3 (2017) que exige $30 \%$ de membros independentes no conselho, para adesão ao Programa Destaque em Governança de Estatais, e com a Lei 13.303/16 (BRASIL, 2016), a qual exige mínimo de $25 \%$. Entretanto, das 12 sociedades de economia mista selecionadas na amostra, apenas 6 apresentaram proporção de membros independentes maior que $30 \%$.

Tabela 3 Estatísticas descritivas

\begin{tabular}{c|c|c|c}
\hline Variável & Média & Desvio-padrão & Observações \\
\hline VOL & 0,027935 & 0,0088760 & 34 \\
\hline TAMC & 2,134211 & 0,2561333 & 34 \\
\hline MUL & 0,093706 & 0,0894432 & 34 \\
\hline INDP & 0,307131 & 0,2251563 & 34 \\
\hline TAMF & 17,650026 & 1,7366467 & 34 \\
\hline ROA & 0,043647 & 0,0970273 & 34 \\
\hline DIV & 0,636298 & 0,2113860 & 34 \\
\hline
\end{tabular}

Nota. A definição das variáveis é dada por: VOL (logaritmo natural da razão entre cotação de fechamento do dia t e t-1); TAMC (logaritmo natural do tamanho do conselho); MUL (proporção de mulheres no conselho); INDP (proporção de membros independentes no conselho); TAMF (logaritmo natural do tamanho da firma); ROA (retorno sobre o ativo); DIV (razão entre dívida total e ativo total). 
Verificou-se, também, que 4 empresas da amostra não estavam listadas nos Níveis Diferenciados de Governança Corporativa (NDGC) durante o período analisado, sendo que a Petrobras iniciou sua listagem no Novo Mercado apenas em dezembro de 2017. Vale ressaltar que a maioria das empresas listadas no NDGC pertencem ao Nível 1, com menor nível de exigência em relação às práticas de governança corporativa.

Partindo da análise descritiva para a análise de regressão, verificou-se, inicialmente, se o modelo atendia a alguns critérios para a realização da regressão linear múltipla, como ausência de multicolinearidade, homocedasticidade e distribuição normal dos resíduos. Verificou-se a ausência de multicolinearidade entre as variáveis independentes, através da análise de correlações e estatísticas de colinearidade, especificamente, pelo Variance Inflation Factor (VIF). A partir dos resultados do VIF menores que 10, para cada variável, verificou-se que há ausência de multicolinearidade. O maior valor de VIF encontrado foi 3,311. Também verificou-se ausência de forte correlação entre as variáveis independentes, conforme apresenta a Tabela 4.

Os pressupostos de homocedasticidade e distribuição normal dos resíduos foram verificados através do gráfico de dispersão dos resíduos. Adicionalmente, foi verificada a distribuição normal dos resíduos por meio do teste não-paramétrico Komolgorov-Smirnov, que apresentou um $p$-value $=0,161$.
$\mathrm{O}$ modelo de regressão apresentou $\mathrm{R}^{2}$ igual a 0,251 , indicando que $25,1 \%$ de variação na variável de volatilidade é explicada pelo modelo. Verifica-se, a partir da Tabela 5, que as variáveis tamanho do conselho e tamanho da empresa apresentam relação positiva, porém não significativa com a volatilidade. Por sua vez, as variáveis proporção de mulheres, proporção de membros independentes, ROA, endividamento total, e adesão ao NDGC apresentam relação negativa, embora não significativa, com a variável volatilidade.

Em relação ao tamanho do conselho, os resultados da regressão apontam para uma relação positiva e não significativa com a volatilidade da cotação das ações. Nesse caso, pode-se evidenciar o argumento de Jensen (1993) de que conselhos maiores apresentam problema de coordenação. A relação encontrada diverge dos resultados obtidos por Cheng (2008), Huang e Wang (2015) e Mathew, Ibrahim e Archbold (2016). Cheng (2008) também utilizou o logaritmo do tamanho do conselho e risco mensurado pelo desvio padrão dos retornos mensais das ações. Huang e Wang (2015) consideraram o número de membros no conselho e risco pelo logaritmo da variância de retornos mensais das ações em 12 meses. Mathew, Ibrahim e Archbold (2016), também consideraram o logaritmo do tamanho do conselho e utilizaram as variáveis de risco total e volatilidade do retorno dos ativos.

Tabela 4 Matriz de correlação

\begin{tabular}{c|c|c|c|c|c|c|c|c}
\hline & VOL & TAMC & MUL & INDP & TAMF & ROA & DIV & NDGC \\
\hline VOL & 1,000 & & & & & & & \\
\hline TAMC & $0,0337^{* *}$ & 1,000 & & & & & & \\
\hline MUL & $-0,079$ & $-0,243^{*}$ & 1,000 & & & & & \\
\hline INDP & $-0,004$ & 0,105 & $-0,23$ & 1,000 & & & & \\
\hline TAMF & $0,237^{*}$ & 0,171 & $-0,179$ & $0,414^{*}$ & 1,000 & & & \\
\hline ROA & $-0,271^{*}$ & $-0,437$ & $0,452^{* *}$ & $-0,131$ & $-0,384$ & 1,000 & & \\
\hline DIV & $-0,006$ & 0,043 & $-0,394^{* *}$ & $-0,122$ & $0,526^{* *}$ & $-0,523^{* *}$ & 1,000 & \\
\hline NDGC & 0,117 & 0,257 & 0,198 & 0,016 & 0,024 & $0,230^{*}$ & $-0,456^{* *}$ & 1,000 \\
\hline
\end{tabular}

Nota. A definição das variáveis é dada por: VOL (logaritmo natural da razão entre cotação de fechamento do dia t e t-1); TAMC (logaritmo natural do tamanho do conselho); MUL (proporção de mulheres no conselho); INDP (proporção de membros independentes no conselho); TAMF (logaritmo natural do tamanho da firma); ROA (retorno sobre o ativo); DIV (razão entre dívida total e ativo total). * valores significativos a $10 \% ;{ }^{* *}$ valores significativos a $5 \%$. 
Tabela 5 Resultados da regressão para a relação entre atributos do conselho e risco

\begin{tabular}{c|c}
\hline Variáveis & Volatilidade (VOL) \\
\hline TAMC & $\begin{array}{c}0,196 \\
(0,935)\end{array}$ \\
\hline MUL & $\begin{array}{c}-0,007 \\
(-0,038)\end{array}$ \\
\hline INDP & $\begin{array}{l}-0,305 \\
(-1,374)\end{array}$ \\
\hline TAMF & $\begin{array}{c}0,462 \\
(1,682)\end{array}$ \\
\hline ROA & $\begin{array}{c}-0,273 \\
(-1,131)\end{array}$ \\
\hline DIV & $\begin{array}{c}-0,430 \\
(-1,428)\end{array}$ \\
\hline NDGC & $-0,01$ \\
& $(-0,006)$ \\
\hline Obs. & 34 \\
\hline
\end{tabular}

Nota. A definição das variáveis é dada por: VOL (logaritmo natural da razão entre cotação de fechamento do dia t e t-1); TAMC (logaritmo natural do tamanho do conselho); MUL (proporção de mulheres no conselho); INDP (proporção de membros independentes no conselho); TAMF (logaritmo natural do tamanho da firma); ROA (retorno sobre o ativo); DIV (razão entre dívida total e ativo total). Valores da estatística-t entre parênteses.

Os resultados indicam, ainda, uma relação negativa não significativa entre proporção de mulheres no conselho e a volatilidade das ações das estatais listadas. A relação evidencia que uma maior presença de mulheres nos conselhos está associada a uma diminuição no risco de mercado, mensurado pela volatilidade da cotação das ações. Tal resultado está em concordância com resultados encontrados por Mathew, Ibrahim e Archbold (2016) e Bernile, Bhagwat e Yonker (2018), considerando diferentes medidas de risco, baseadas na volatilidade do retorno das ações. A relação encontrada pode ser explicada no sentido expresso por Bernile, Bhagwat e Yonker (2018) de que a diversidade atua como um mecanismo moderador das decisões.

Verifica-se, ainda, que a independência dos membros do conselho também apresenta relação negativa não significativa, com a volatilidade, de modo que maior proporção de conselheiros independentes diminui o risco de mercado para as estatais listadas. Tal resultado está em concordância com a relação encontrada por Cheng (2008), que também encontrou uma relação não significativa, e por Mathew, Ibrahim e Archbold (2016).

Em relação às variáveis de controle, verifica-se que apenas o tamanho da empresa apresentou relação positiva com a variável de risco, sendo que todas apresentaram relações não significativas estatisticamente. A relação das variáveis tamanho da empresa e endividamento com a variável de risco teve resultado contrário do esperado com base na literatura. Por outro lado, a variável relativa à adesão das empresas aos níveis diferenciados de governança corporativa da B3 apresentou relação negativa, mas não significativa, com o risco, em concordância com a literatura, que evidencia maior transparência, segurança e redução de riscos para as empresas que aderirem a estes segmentos especiais de listagem. A variável de retorno sobre o ativo também apresentou relação negativa, mas não significativa, com a variável de risco, o que indica que melhor desempenho financeiro está associado a menor risco.

Diante dos resultados apresentados pela regressão, é possível compreender a direção do relacionamento entre determinadas características dos conselhos de administração - tamanho e proporção de mulheres e conselheiros independentes - e o risco de mercado, mensurado pela volatilidade da cotação das ações, apresentado pelas estatais listadas analisadas.

Entretanto, nota-se que as variáveis relativas a atributos do conselho não apresentaram relações estatisticamente significativas com a variável de risco. Sendo assim, é possível que existam efeitos, não capturados nas variáveis, que exercem influência sobre o risco de mercado apresentado por tais empresas. Também é possível destacar, a partir dos resultados da regressão, que boas práticas de governança nos conselhos - número adequado de membros, proporção de mulheres e membros independentes - pode não ser eficiente para minimizar a volatilidade do preço das ações das estatais analisadas. Ademais, cabe ressaltar que é difícil constatar, de fato, a independência de um conselheiro no âmbito das estatais, uma vez que tais indivíduos podem esconder afiliações políticas (MENOZZI; URTIAGA; VANNONI, 2012). 


\section{CONSIDERAÇÕES FINAIS}

O presente estudou buscou investigar a relação entre características dos conselhos de administração de estatais brasileiras listadas e seu risco de mercado. Os resultados apontam que o tamanho dos conselhos das estatais apresenta relação positiva não significativa com a volatilidade da cotação das ações, o que poderia evidenciar possíveis conflitos de coordenação em conselhos maiores. Por outro lado, a proporção de mulheres e de membros independentes no conselho apresenta relação negativa com a volatilidade da cotação das ações, em concordância com resultados encontrados na literatura, no entanto, tal relação não é significativa.

Embora seja possível compreender a direção das relações entre as variáveis relativas ao conselho e ao risco, tais relações não são estatisticamente significativas. Dessa forma, considerando as particularidades das empresas estatais, é possível que alguns efeitos não capturados pelas variáveis, exerçam influência sobre o risco de mercado de tais empresas. Do mesmo modo, o estudo evidencia indícios de que a governança corporativa não seja eficiente para minimizar o risco, mesmo com a adesão da maioria das empresas analisadas ao NDGC, mesmo que no Nível 1, com menor nível de exigências em relação à governança corporativa. A declaração de independência de determinados conselheiros pode cumprir as exigências da legislação, porém, pode esconder afiliações políticas mantidas de forma indireta, prejudicando a autonomia do conselho para exercer sua função de monitoramento. Ademais, os conselhos de administração de empresas estatais ainda carecem de regulações mais específica em relação à inclusão de mulheres de forma efetiva.

Recomenda-se, para estudos futuros, considerar a estrutura do conselho de administração de forma mais ampla para avaliar seu efeito em diferentes variáveis de risco das estatais, além de realizar uma comparação com conselhos de empresas privadas. Em adição, é possível analisar se as mudanças previstas na Lei de Responsabilidade das Estatais, Lei Federal $\mathrm{n}^{\circ} 13.303$ de 2016, foram adotadas pelos conselhos das estatais e se houve impacto no risco de mercado de tais empresas.
O presente estudo contribui para maior entendimento da influência que o conselho de administração tem sobre o risco das empresas de controle estatal listadas, que atuam em diferentes setores estratégicos da economia. Ademais, tal entendimento é importante, considerando que uma parcela de tais empresas compõe o Ibovespa, além de capitalizarem grande volume de recursos. Entretanto, apresenta-se como limitação à pesquisa o número reduzido de estatais listadas com dados disponíveis para compor as variáveis consideradas neste estudo.

Em termos organizacionais, o estudo chama a atenção para o panorama atual dos conselhos de administração das estatais listadas no Brasil, evidenciando que o tamanho de determinados conselhos pode não ser adequado e, principalmente, que é preciso promover maior diversidade de gênero nestes órgãos. Em termos acadêmicos, contribui-se ao ampliar a literatura, ainda limitada, sobre governança corporativa de empresas estatais no Brasil, contexto que apresentou ações recentes, do mercado e da legislação, para aprimorar a governança dessas empresas, evidenciando a relevância de tal discussão. Em adição, o estudo mostra a necessidade de se discutir a efetividade das formas atuais de capitalismo de estado no Brasil, considerando que as estatais listadas lidam com objetivos múltiplos ao oferecer serviços em benefício da sociedade, ao mesmo tempo em que maximizam valor para seus acionistas.

\section{AGRADECIMENTOS}

O presente trabalho foi realizado com apoio da Coordenação de Aperfeiçoamento de Pessoal de Nível Superior - Brasil (CAPES) - Código de Financiamento 001 .

\section{REFERÊNCIAS}

ADAMS, R. B.; FERREIRA, D. Women in the boardroom and their impact on governance and performance. Journal of Financial Economics, v. 94, n. 2, p. 291-309, 2009. 
ALLINI, A.; ROSSI, F. M.; HUSSAINEY, K. The board's role in risk disclosure: an exploratory study of Italian listed state-owned enterprises. Public Money \& Management, v. 36, n. 2, p. 113-120, 2016.

ARAÚJO, D. L et al. O risco de mercado do agronegócio brasileiro: uma análise comparativa entre os modelos CAPM e GARCH-M. Revista Eletrônica de Gestão Organizacional, v. 2, n. 3, p. 207-220, 2004.

ASSAF NETO, A. Finanças corporativas e valor. 5a ed. São Paulo: Atlas, 2010.

B3. Regimento do Programa Destaque em Governança de Estatais. 2017. Disponível em: <http://www.bmfbovespa.com.br/pt_br/listagem/ acoes/governanca-de-estatais/>Acesso em: $14 \mathrm{dez}$. 2017.

BERLE, A.; MEANS, G. The modern corporation and private property. New Brunswick: Transaction Publishers, 1932.

BERNILE, G.; BHAGWAT, V.; YONKER, S. Board diversity, firm risk, and corporate policies. Journal of Financial Economics, v. 127, n. 3, p. 588-612, 2018.

BRASIL. Lei ${ }^{\circ} 13.303$, de 30 de junho de 2016 . Dispõe sobre o estatuto jurídico da empresa pública, da sociedade de economia mista e de suas subsidiárias, no âmbito da União, dos Estados, do Distrito Federal e dos Municípios. Diário Oficial da União. Brasília, DF, 01 jul. 2016. Seção 1. Disponível em: <http:// www2.camara.leg.br/>. Acesso em: 10 mai. 2018.

CHARNESS, G.; GNEEZY, U. Strong Evidence for Gender Differences in Risk Taking. Journal of Economic Behavior \& Organization, v. 83, n. 1, p. 50-58, 2012.

CHENG, S. Board size and the variability of corporate performance. Journal of Financial Economics, v. 87, n. 1, p. 157-176, 2008.

CHRISTIE, A. A. The stochastic behavior of common stock variances. Journal of Financial Economics, v. 10, n. 4, p. 407-432, 1982.
COLETTA, C.; LIMA, R. A. S. Board of directors, performance and firm value in Brazilian listed state-owned enterprises. Brazilian Review of Finance, v. 18, n. 2, p. 1-28, 2020.

COSTA, H. U.; MAZZEU, J. H. G.; COSTA Jr., N. C. A. O comportamento dos componentes da volatilidade das ações no Brasil. Revista Brasileira de Finanças, v. 14 , n. 2, p. 225-268, 2016.

IBGC. Boas práticas de governança corporativa para sociedades de economia mista. Série Cadernos de Governança Corporativa. São Paulo: IBGC, 2015a.

Código das melhores práticas de governança corporativa. 5a. ed. São Paulo: IBGC, 2015 b.

Boas práticas de governança corporativa para empresas estatais. São Paulo: IBGC, 2020.

FAMA, E. F.; JENSEN, M. C. Separation of ownership and control. Journal of Law and Economics, v. 26, n. 2, p. 301-325, 1983.

HUANG, Y. S. WANG, C. J. Corporate governance and risk-taking of Chinese firms: the role of board size. International Review of Economics and Finance, v. 37, p. 96-113, 2015.

JENSEN, M. C. The modern industrial revolution, exit, and the failure of internal control systems. Journal of Finance, v. 48, n. 3, p. 831-880, 1993.

JENSEN, M. C.; MECKLING, W. H. Theory of the firm: managerial behaviour, agency costs and ownership structure. Journal of Financial Economics, v. 3, p. $305-360,1976$.

KHONGMALAI, O.; DISTANONT, A. Corporate governance model in Thai state-owned enterprises: structural equation modelling approach. Corporate Governance: The International Journal of Business in Society, v. 17, n. 4, p.613-628, 2017. 
LAMEIRA, V. J. As relações entre governança e risco nas companhias abertas brasileiras. Revista Brasileira de Gestão de Negócios, v. 14, n. 42, p. 7-25, 2012.

MATHEW, S.; IBRAHIM, S.; ARCHBOLD, S. Boards attributes that increase firm risk - evidence from the UK. Corporate Governance: The International Journal of Business in Society, v. 16, n. 2, p. 233258,2016

Corporate governance and firm risk. Corporate Governance: The International Journal of Business in Society, v. 18, n. 1, p. 52-67, 2018.

MATIAS, A. B. Finanças corporativas de longo prazo: criação de valor com sustentabilidade financeira. São Paulo: Atlas, 2010, 2 v.

MENOZZI, A.; URTIAGA, M. G.; VANNONI, D. Board composition, political connections, and performance in state-owned enterprises. Industrial and Corporate Change, v. 21, n. 3, p. 671-698, 2012

MIRANDA, R. A.; AMARAL, H. F. Governança corporativa e gestão socialmente responsável em empresas estatais. Revista de Administração Pública, v. 45, n. 4, p. 1069-1094, 2011.

MUSSACHIO, A.; LAZZARINI, S. G.; AGUILERA, R. V. New varieties of state capitalism: strategic and governance implications. The Academy of Management Perspectives, v. 29, n. 1, p. 115-131, 2015.

NAKANO, M.; NGUYEN, P. Board size and corporate risk taking: further evidence from Japan. Corporate Governance: An International Review, v. 20, n. 4 , p. 369-387, 2012.

OECD. OECD Guidelines on corporate-governance of state-owned enterprises. 2nd ed. Paris: OECD Publishing, 2015.
PAIVA, J. F. M.; OLIVEIRA, N. A.; PEIXOTO, F. M. A relação entre conselho de administração, desempenho, valor e risco no mercado brasileiro de ações. Revista de Educação e Pesquisa em Contabilidade, v. 9, n. 1, p. 25-44, 2015.

POWELL, M.; ANSIC, D. Gender differences in risk behaviour in financial decision-making: an experimental analysis. Journal of Economic Psychology, v. 18, n. 6, p. 605-628, 1997.

SHLEIFER, A.; VISHNY, R. W. Politicians and firms. The Quarterly Journal of Economics, v. 109, n. 4, p. 995-1025, 1994.

SHI, H.; XU, H.; ZHANG, X. Do politically connected independent directors create or destroy value? Journal of Business Research, v. 83, p. 82-96, 2018.

SILA, V.; GONZALEZ, A.; HAGENDORFF, J. Women on board: Does boardroom gender diversity affect firm risk?. Journal of Corporate Finance, v. 36, p. 26-53, 2016.

SILVA, R. L. M; NARDI, P. C. C.; PIMENTA JUNIOR, T. O impacto da migração das empresas para os níveis diferenciados de governança corporativa da $\mathrm{BM} \& \mathrm{~F}$ Bovespa sobre o risco e o retorno de suas ações. Revista de Administração da UFSM, v. 5, n. 2, p. 222-242, 2012.

SILVEIRA, A. D. M. Governança corporativa no Brasil e no mundo: teoria e prática. Rio de Janeiro: Elsevier, 2010.

THOMPSON, R. M., ALLEYNE, P.; CHARLESSOVERALL, W. Exploring governance issues among boards of directors within state-owned enterprises in Barbados, International Journal of Public Sector Management, v. 32, n. 3, p. 264-281, 2019.

YERMACK, D. Higher market valuation of companies with a small board of directors. Journal of Financial Economics, v. 40, n. 2, p. 185-211, 1996. 